interpreted as the time the spread of a vibratory wave or a ball movement takes to match the given distance. But then the content of the second product term of Equation 1 cannot be reduced to matching. This becomes obvious from the fact that the $d$ range enters for the ball data the same way as for the weight data. Furthermore, there is no effect of absolute time. Rather, it enters into the first (distance independent) and into the second term relative to matching time.

The limited data base does not warrant a detailed discussion of the parameter constraints per se. A possible exception is the decay parameter of 8.1 seconds, which obviously depends on autonomous characteristics of the processing systems. It may be noted that there is a significant body of evidence from various paradigms of a universal critical time period of about 9 seconds (cf. Geissler 1987; 1991).

Rule constraints and optimality. In addition to parametric constraints the example illustrates the presumptive existence of a complementary type of inner constraint on cognition that may be called a rule constraint. This involves limitations of task adaptivity caused by the format of potential rules. As causality judgments are a particular case of information integration in the sense defined by N. H. Anderson, rule (1) can be looked upon as a complex "mental algebra" (cf., e.g., N. H. Anderson 1981). A first argument suggesting that this classification is useful comes from the empirical evidence that complex rules result from combining a small number of elementary rules. Thus a major goal of RA, the derivation of cognitive rules, could be related to an inner iterative process of rule construction.

A second argument relates rule constraints to optimality. An example bearing on sensation is given in Geissler and Puffe (1983). The general argument is as follows. Suppose some set of constraints among subjective variables is modelled by a set of rule constraints among subjective variables. Then, in general, both sets of constraints do not uniquely determine the mappings of objective variables onto subjective ones. Uniqueness can be attained by assuming extremality principles as a basis for selection rules. For sensory attributes these principles take the form of invariance or constancy predicting power laws of mapping for multiplicative, and logarithmic laws for additive rule constraints. In the realm of perceptual organization the same basic principle takes the form of the minimum principle of structural information (cf. Leeuwenberg \& Buffart 1983).

Extremality principles of this type are forms of optimality criteria that seem to have nothing to do with those advocated by Anderson. On a deeper level, however, at least two relationships between both types of criteria can be relevant within a broader theoretical framework: (1) Cognitive performance can become optimal in the sense of an absolute structural minimum in a stationary state after the application of Anderson's rule. This may be trivial in some problem-solving tasks where the solver proceeds along a trajectory of minimal length from the start to the goal after recognizing the general solution. Minimality reached in this way can be nontrivial in other cases, for example, if the task of cognition is to find a shortest cognitive code of a category. (2) A cognitive code may involve a hierarchy, with both types of optimal criteria operating. A memory search task, for example, may be accomplished using the optimal memory code at hand.

Anderson's theory of categorization presupposes well-defined and fixed features. (2) will become relevant in situations in which features must be considered the result of structure formation processes that become part of adaptive behavior. This expanded notion of adaptivity may also apply to the assimilation of category structures. Geissler and Puffe (1983) and Buffart and Geissler (1983) provide evidence that the phenomenon of basic level categories, which is considered primary by Rosch and coworkers, Hoffmann and Ziessler (1983) and others, can be derived from a generalized minimum principle.

The place of Bayesian statistics. The above considerations can be summarized as follows: (a) The deductive something of RA and more inductive strategies of analysis may represent complementary exploratory paths rather than mutually exclusive ones. (b) It seems useful to complement the rationale of RA by techniques which take into account inner constraints on brain activity or, if you like, the "structure of mind." (c) RA and AV become related theoretical points of view as soon as the representation and generation of information become mutually dependent.

If this is true, what about Bayes' theorem? To me it seems a very forceful implementation of Helmholtz's principle of unconscious or inductive inference. It has turned out to be of great heuristic value in the prediction of visual illusions within the indirect validation framework I used in early work (Geissler 1970). Still, I suspect it needs modifying if inner constraints are implied. If a guess is permitted, it might take the form of a selfconsistency relation established within neuronal networks of the types suggested by the principles of adaptive resonance (Grossberg 1988) or reentry (Edelman 1987).

\section{Does the environment have the same structure as Bayes' theorem?}

\section{Gerd Gigerenzer}

Institut für Psychologie, Universität Salzburg, 5020 Salzburg,

Hellbrunnerstraße 34, Austria

Electronic mail: 1gigerenz@edvz.uni-salzburg.ada.at

Cognition should not be divorced from its environment, argued Egon Brunswik (1964), comparing the two to a married couple who have to come to terms with one another by mutual adaptation. His "ratiomorphic explication" of cognition started with analyzing the statistical texture of the natural environments (the ecological validities) and the degree to which perception is adapted to that texture. Anderson's program of "rational analysis" is quite similar: To specify the statistical structure of the environment, and, on the assumption that cognition is adapted to that structure, to infer the structure of cognition - or, at least, to infer constraints imposed on cognition by the environment Both Brunswik and Anderson study the coming-to-terms of the married couple as an adaptation of only one partner (cognition) to the other, and both view the mind as an intuitive statistician. But here the similarities end.

Does Anderson pursue his own program? The crucial Step 2 of the "rational analysis" is "to specify the structure of the environment to which cognition is optimized," which "is much easier to observe than the structure of the mind." How do we observe that environmental structure? Among three approaches, Anderson proposes the "appeal to existing scientific theory" as the most compelling, to be illustrated with a rational analysis of categorization. So let us look at that: What is the structure of the environment that is reflected in the structure of category formation? Anderson proposes two structural components, the disjoint partitioning of the object set, and the independence of features of objects. Both are necessary assumptions for his Bayesian modelling of categorization and other cognitive functions. In the case of categorization, the evolutionary rationale Anderson gives is twofold: (1) that species cannot interbreed (disjoint partitioning of the object set), and (2) that features within species are displayed largely independently of one another.

Even if these two structural components were characteristic for the evolutionary context - Anderson himself admits that independence does not hold when features are controlled by the same gene - the question is whether they are characteristic for other contexts, too, as Anderson assumes. Conditional independence is a mathematically convenient assumption in standard 
Bayesian models, but not necessarily valid in natural environments. Brunswik in fact focused on the dependencies between features of objects in natural environments, which for him defined the texture of an environment. Similarly, physicians look for clusters of dependent symptoms to arrive at a disease classification. In general, conditional nonindependence among testimonial evidence, clinical symptoms, and other features poses a well-known problem in the sequential application of Bayes' rule, as it does in Anderson's "rational theory." Dependence between the prior probability and the likelihood ratio (which measures the impact of new information) in Bayes' theorem poses another problem (e.g., Birnbaum 1983).

This may be sufficient to illustrate why I do not think that Anderson pursues his own program: to analyze the structure of the environment. Rather, he seems to have started with Bayes' theorem as a model of rationality and to have assumed that the structural assumptions underlying Bayes' theorem specify the structure of the environment as well. This is a legitimate heuristic: to start with some statistical model of inference Fisher's analysis of variance, Neyman-Pearson decision theory, multiple regression, Bayes' theorem - and to investigate the hypothesis that the mind is an intuitive statistician of that kind or school (Gigerenzer 1991). And this is what I understand Anderson to be doing. But this is not Anderson's program according to his own lights.

is Bayesian statistics adaptive? "The information-processing implications of various environmental cues are not certain. This fact leads to the Bayesian character of the optimization analysis. . . "Why? The same fact leads Brunswik to the multipleregression character of optimization. Neither Brunswik nor Anderson explains why they believe that their respective statistics would be adaptive. Bayesianism seems to be flexible enough to apply to any environment, even to those commonly seen to contradict it, such as Allais's and Ellsberg's paradoxes (e.g., Jeffrey 1987). But insofar as there is a specific Bayesian model of some cognitive function, I believe that the question whether the model applies to a given environment can be answered only empirically, not a priori. This can be done by checking whether a structural isomorphism exists between a given environment (or task) and the specific Bayesian model (see Gigerenzer \& Murray 1987, pp. 162-74). Similarly, if we want to see the mind as a rational intuitive statistician (Bayesian or otherwise), then we need to postulate not only a statistical algorithm, but in addition some heuristics (or a second-order algorithm) that check whether the structural assumptions of the algorithm hold in the given environment over time and space.

Toward domain-specific theories of cognition. One direction for revising the rational analysis would be to change the singular form "to specify the structure of the environment" (Step 2) into the plural form "to specify the structures of environments." Different environments may have different structures, and these may also change over time. Thus, the program would need an extra step before Step 2 to obtain a categorization of various environments. Let us call the product of this categorization a set of domains. Domains may correspond with respect to level of abstraction and predictive power to Rosch's basic level objects (e.g., Rosch 1978). For example, the recent proposal of domainspecific theories of reasoning has greatly advanced the potential to predict people's information search in the Wason selection task (e.g., Cheng \& Holyoak 1985; Cosmides 1989). Proposed domains (of human interaction) include social contracts, threats, permissions, obligations. In a social contract, for example, a decisive structural component seems to be that a participant can be cheated and that subjects consistently search for information that can reveal potential cheaters. [See Maynard Smith: "Game Theory and the Evolution of Behaviour" $B B S$ 7(1) 1984; and Caporael et al.: "Selfishness Examined: Cooperation in the Absence of Egoistic Incentives" BBS 12(4) 1989.]

Bayesian models can indeed be very useful in suggesting a conceptual language for talking about differences in structures across domains. But domains also have surplus structures, such as cheating options, which go beyond standard statistical structures. If we take Anderson's program seriously and start with a theory of environments (as opposed to starting with Bayes' theorem) then we might indeed make the "substantial discovery" that Anderson promises. But we might also discover that cognition is more flexible and does not always rely on Bayes' theorem and strong assumptions such as independence. A highly adaptive intuitive statistician of the mind might even work with exploratory data analysis.

\section{Optimality and psychological explanation}

\section{Peter Godfrey-Smith}

Museum of Comparative Zoology, Harvard University, Cambridge, MA $02138^{1}$

Anderson seeks to apply optimality methods to cognition without appealing to a biological justification for this approach, claiming that the optimality framework should simply "stand on its own feet in accounting for data." The program that results, according to Anderson, is a "high-risk, high-gain enterprise" compared to standard approaches to cognitive psychology. That this strategy is high-risk is quite certain; its promise of high gains is another matter. The main issue $I$ discuss is whether the optimality approach, removed from its biological context, is in principle able to deliver the explanatory payoff Anderson seeks.

We must distinguish two kinds of explanatory success that any approach to cognition might deliver. First, there is what Anderson refers to as "organizing the data." A theory might produce impressive generalizations and predictions about actual cognitive phenomena, leading us to believe that it gives us an accurate account of the actual structure of the mind. But once we have a good understanding of how the mind is wired up, we should be led to a second question: Why is the mind wired the way it is? These questions are quite distinct, though it is possible for them to be investigated at the same time and with similar methods.

Optimality theory is controversial and high risk, but it may appear that the risks are offset by the possibility that this approach can yield both kinds of explanatory success. Very possibly, this is how things appear to Anderson, who thinks that as well as organizing the data, his approach takes seriously "the idea that there is reason for the way the mind is." The problem with Anderson's approach is that severing optimality from biology robs the optimality approach of its ability to yield insight into this mental raison d'être. This is because it is only as a component within a more general biological approach that optimality has this kind of explanatory potential.

Before I enlarge on this, it is important to recognize that Anderson might choose to claim only the first kind of explanatory role for his "rational analysis." That is, the sole purpose might be to describe the actual structure of cognition, making no claims to explain why we are structured the way we are. I do not discuss this view of Anderson's target article in detail. I am skeptical about his specific models but provide motivation for this skepticism with one brief comment only. It is unlikely that an analysis of some aspect of cognition in terms of costs and benefits will succeed if the only costs considered are those "internal" costs deriving from the mental effort involved in retrieving memories, forming hypotheses, and the like (Table 2). Such costs must surely pale beside practical, external costs resulting from bad behavioral choices. To some extent, internal costs are related to the external, practical ones. Expending "internal effort" is practically costly if it also spends time, for example, and the mental operations Anderson considers may monopolize the resources of attention in critical decision-making situations. But if Anderson thinks internal effort has signifi- 